Astrobiology 18, 897-914 (2018).

3. Fry, I. in Handbook of Astrobiology (ed. Kolb, V. M.) Ch. 3.1, 109-124 (CRC, 2019).

4. Pascal, R., Pross, A. \& Sutherland, J. D. Open Biol. 3 , 130156 (2013)
5. Sutherland, J. D. Nature Rev. Chem. 1, 0012 (2017).

6. Bruylants, G., Bartik, K. \& Reisse, J. C. R. Chim. 14, 388-391 (2011)

7. Ruiz-Mirazo, K., Briones, C. \& de la Escosura, A. Chem. Rev. 114, 285-366 (2014).
8. Miller, S. L. Science 117, 528-529 (1953).

9. Lotka, A. J. Proc. Natl Acad. Sci. USA 8, 151-154 (1922).

10. Pross, A. What is Life? How Chemistry Becomes Biology (Oxford Univ. Press, 2016).

\title{
A doubly magic nucleus that has two faces
}

The neutron-rich nickel nucleus ${ }^{78} \mathrm{Ni}$ is difficult to excite and, once excited, has competing spherical and deformed shapes. These intriguing properties make ${ }^{78} \mathrm{Ni}$ a valuable testing ground for nuclear theory. SEE ARTICLE P.53

\section{GAUTE HAGEN \& THOMAS PAPENBROCK}

$\mathrm{N}$ ot all atomic nuclei are created equal. 'Doubly magic' nuclei have fully occupied shells of protons and neutrons, the subatomic particles known generically as nucleons. Such nuclei are therefore more strongly bound together and more difficult to excite than their neighbours on the Segrè chart - a 2D grid in which nuclei are arranged by their proton and neutron numbers. The simple structure of doubly magic nuclei makes them the cornerstone of our understanding of nuclear physics. This is because their neighbours can be described in terms of a few extra interacting nucleons, therefore turning a quantum many-body problem into a fewbody problem. Only a handful of doubly magic nuclei are known. On page 53, Taniuchi et al. ${ }^{1}$ identify the newest member of this elite club: the nickel nucleus ${ }^{78} \mathrm{Ni}$, which consists of 28 protons and 50 neutrons.

Naturally occurring nickel - used in coins, rechargeable batteries and plumbing fixtures - typically has only 30 neutrons in its nucleus. By comparison, ${ }^{78} \mathrm{Ni}$ is extremely rich in neutrons, which makes it difficult to produce and study. This rare isotope of nickel was first observed ${ }^{2}$ in 1995. Apart from its halflife, which was measured ${ }^{3}$ in 2005 to be only about 0.1 seconds, little was known about its properties.

Taniuchi and colleagues made and investigated ${ }^{78} \mathrm{Ni}$ by knocking one and two protons out of beams of rare isotopes of copper $\left({ }^{79} \mathrm{Cu}\right)$ and zinc $\left({ }^{80} \mathrm{Zn}\right)$, respectively. These beams were produced at the Radioactive Isotope Beam Factory in Wako, Japan. Coinciding with the proton-knockout reactions, the authors observed $\gamma$-rays that were emitted when ${ }^{78} \mathrm{Ni}$ transitioned from various excited states to the ground state. Key challenges that Taniuchi et al. had to contend with included low rates of ${ }^{78} \mathrm{Ni}$ production from the rareisotope beams and the need to measure a substantial number of associated $\gamma$-rays.

The authors detected a strong emission of $\gamma$-rays when ${ }^{78} \mathrm{Ni}$ transitioned from an excited state at an energy of 2.6 megaelectronvolts (MeV) to the ground state (Fig. 1). The relatively large energy of this excited state identified ${ }^{78} \mathrm{Ni}$ as a doubly magic nucleus. Taniuchi et al. suggest that this excited state is a $2^{+}$state of ${ }^{78} \mathrm{Ni}$ that is associated with a spherical nucleus - the ' 2 ' refers to the intrinsic angular momentum of the nucleus and the '+' indicates that the nucleus has a property known as even parity. This assignment is corroborated by theoretical calculations made by the authors, and by earlier predictions ${ }^{4,5}$.

The identification of ${ }^{78} \mathrm{Ni}$ as a doubly magic nucleus provides us with a beachhead from which to explore neutron-rich nuclei in the vicinity of ${ }^{78} \mathrm{Ni}$. Such exploration is valuable because the synthesis of heavy elements in the

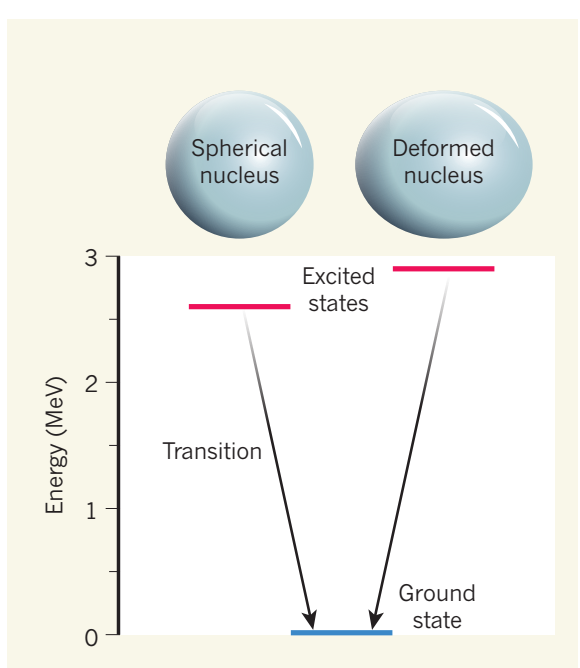

Figure 1 | Properties of the nickel nucleus ${ }^{78} \mathrm{Ni}$. Taniuchi et al. ${ }^{1}$ report transitions of ${ }^{78} \mathrm{Ni}$ between excited states and the ground state. An excited state at an energy of 2.6 megaelectronvolts $(\mathrm{MeV})$ suggests that ${ }^{78} \mathrm{Ni}$ is a type of strongly bound nucleus called a 'doubly magic' nucleus. Furthermore, an excited state with an energy of about $2.9 \mathrm{MeV}$ indicates that excited ${ }^{78} \mathrm{Ni}$ can exist with both spherical and deformed shapes.
Universe proceeds - by means of a nuclear reaction called neutron capture - along pathways that include neutron-rich nuclei that are close to nickel on the Segrè chart. In the neighbourhood of ${ }^{78} \mathrm{Ni}$, a radioactive process known as $\beta$-decay is dominant over neutron capture, which leads to enhanced natural abundances of nuclei that have about 80 nucleons. Taniuchi and colleagues' work will enable nuclear physicists to advance understanding of the neutron-rich nuclei that lie between ${ }^{78} \mathrm{Ni}$ and the next cornerstone on the Segrè chart: the tin nucleus ${ }^{132} \mathrm{Sn}$, which was shown ${ }^{6}$ to be doubly magic in 2010.

Among the other transitions observed in their experiment, Taniuchi et al. tentatively identified a second $2^{+}$state at an energy of about $2.9 \mathrm{MeV}$ that corresponds to a deformed nucleus (Fig. 1). This assignment, which is supported by the authors' theoretical calculations, indicates that excited ${ }^{78} \mathrm{Ni}$ has competing spherical and deformed shapes. The results raise further questions. Is there a deformed $0^{+}$excited state, as predicted by the nuclear-shell model? And why is the spherical $2^{+}$state seen in only the one-proton knockout reaction and the deformed $2^{+}$state seen in only the two-proton reaction?

A coexistence of spherical and deformed shapes is rare in general, but not that unusual in neutron-rich nuclei. It will be interesting to see how these structures change and evolve as a few nucleons are added or removed from ${ }^{78} \mathrm{Ni}$. The competition of the spherical and deformed shapes in ${ }^{78} \mathrm{Ni}$ suggests that nuclei in this region of the Segrè chart - such as neutron-rich isotopes of copper or iron - will be deformed in their ground states. In this sense, ${ }^{78} \mathrm{Ni}$ is a stronghold against deformation in this region. Such a region is called an island of inversion ${ }^{5,7}$ because the usual rules about the structure of nuclei, derived from the nuclearshell model, do not apply.

Investigating and understanding this island of inversion is an exciting yet challenging prospect for nuclear theory and its associated experiments. Deformation sets in when protons and neutrons can occupy spherical shells that are extremely close in energy. In neutronrich nuclei, the nucleons in the corresponding shells are weakly bound to the nucleus, or even unbound. As a result, accurate modelling is required, for instance, to discover the limits of the nuclear landscape - in other words, to determine the maximum number of neutrons that can be bound together by the strong nuclear force in the isotopes of a given chemical element ${ }^{8}$.

For the purposes of theory, it is important to account for nuclear rotation - a collective motion of an entire nucleus. However, 


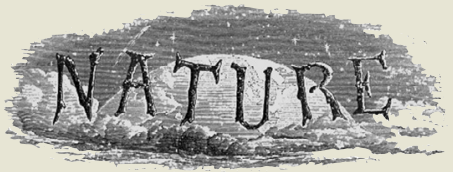

50 Years Ago

The Leningrad astronomer Dr Nikolai Kozyrev has made another remarkable spectrographic observation of the Moon's vulcanism, according to Novosti. It purports to link earthquakes on the Earth, particularly in the United Arab Republic and off the coast of Japan on March 31, with the venting of gases from the Aristarchus crater on the Moon on the following day (April 1). Dr Kozyrev is said to hold that this shows that the Moon "responded" to the earthquakes. Dr Kozyrev is also quoted as saying: "My investigations dealt with the Aristarchus crater. Two spectrograms of the inner slope of the crater ... showed an unusual red spot approximately one to two $\mathrm{km}$ across ... after measurements of the spectrograms obtained by the Crimean observatory, it was established for the first time that this spot is the result of an escape of gases - molecular nitrogen and cyanic gas."

From Nature 3 May 1969

100 Years Ago

A snowstorm of unusual severity ... occurred generally over the British Isles on Sunday, April 27, and in the south-east of England the storm was particularly severe ... In London snow commenced at about 1 p.m. after somewhat heavy rain, and it thoroughly covered the ground by 3 p.m. The depth of snow by the early morning of April 28 in the north of London was $12 \mathrm{in}$. to $15 \mathrm{in}$.... The snowfall occasioned much dislocation of the telegraph and telephone services, and the rapid melting of the snow caused floods in many parts. Temperature on April 27 was abnormally low for so late in the season, the thermometer in London during the afternoon standing at about freezing point. Agriculturists and fruit-growers have suffered somewhat badly.

From Nature 1 May 1919 describing nuclear rotation is a challenge when one tries to build the nucleus from scratch on the basis of individual nucleons, and when, as in the $a b$ initio computations of the present work, one starts with two-nucleon and threenucleon interactions that were constrained solely from the properties of light nuclei (isotopes of hydrogen and helium). The experimental identification of nuclear deformation also requires the measurement of transitions between the energy levels of a sequence of states called a rotational band. Such measurements need relatively high production rates of rare-isotope beams. Taniuchi and colleagues' work is both a milestone in the Segrè chart and an entrance to a previously unexplored region of it. For theory and experiments, the best is yet to come.
Gaute Hagen is in the Physics Division,

Oak Ridge National Laboratory, Oak

Ridge, Tennessee 37831, USA. Thomas

Papenbrock is in the Department of Physics

and Astronomy, The University of Tennessee,

Knoxville, Tennessee 37996, USA.

e-mails:hageng@ornl.gov; tpapenbr@utk.edu

1. Taniuchi, R. et al. Nature 569, 53-58 (2019).

2. Engelmann, Ch. et al. Z. Phys. A 352, 351-352 (1995).

3. Hosmer, P. T. et al. Phys. Rev. Lett. 94, 112501 (2005).

4. Hagen, G., Jansen, G. R. \& Papenbrock, T. Phys. Rev. Lett. 117, 172501 (2016).

5. Nowacki, F., Poves, A., Caurier, E. \& Bounthong, B. Phys. Rev. Lett. 117, 272501 (2016).

6. Jones, K. L. et al. Nature 465, 454-457 (2010).

7. Warburton, E. K., Becker, J. A. \& Brown, B. A.

Phys. Rev. C 41, 1147-1166 (1990)

8. Erler, J. et al. Nature 486, 509-512 (2012).

\section{Sea animals vulnerable to warming}

The impact of climate change on biodiversity is a pressing concern. A study now combines experimental data with careful modelling to compare the vulnerability to warming of animal species on land and in the ocean. SEE LETTER P.108

\section{ANTHONY J. RICHARDSON \\ \& DAVID S. SCHOEMAN}

1 cologists, conservationists and policymakers are struggling to understand how much of a threat climate change poses to Earth's biodiversity - estimated to be some 3 million to 100 million species ${ }^{1}$ and what to do about that threat. Knowing whether terrestrial or marine species are more vulnerable to climate change, as well as how the risks vary with latitude, could inform the deployment of limited conservation resources, nationally and globally. On page 108, Pinsky et al. ${ }^{2}$ combine robust experimental data with a careful model-based approach to compare the vulnerability of biodiversity to warming across latitudes on land and in the ocean.

There is contradictory evidence about the relative vulnerability to warming of land and ocean animals. Terrestrial species could be at greater risk because they are less able to adapt to new climatic conditions ${ }^{3}$ and because they are exposed to higher extreme temperatures than are ocean-dwelling species. But marine species could be more affected because temperature strongly controls their geographic limits ${ }^{4}$, nutrient supplies ${ }^{5}$ and oxygen availability ${ }^{6}$.

Temperature extremes, rather than average temperatures, are an important determinant of population survival at the warm edge of a species' temperature range ${ }^{7}$. Guided by this evidence, Pinsky et al. calculated the thermal safety margin - defined as the difference between the highest temperature at which an animal can survive (its maximum thermal tolerance) and the maximum body temperature that it will effectively experience under natural conditions - for 387 species of ectothermic animal, which rely on external heat to maintain body temperature. The authors calculated two versions of the thermal safety margin for each species: one for when the animal is fully exposed to heat and one for when it is in a thermal refuge. Terrestrial thermal refuges include microclimates such as shade under a tree or rock, whereas marine thermal refuges comprise deeper, cooler waters.

Pinsky et al. found that there are no thermal safety margins for land-dwelling ectotherms when they have no access to thermal refuges, whereas such margins exist for their oceandwelling counterparts. This suggests that land species might be more at risk from climate change than ocean species are. However, when thermal refuges were taken into account, the situation was reversed, with the thermal safety margins being broader for species on land than for those in the ocean (Fig. 1). This implies that marine species might actually be more at risk.

The authors went on to observe that, when thermal refuges were considered, the thermal safety margins of land species were narrowest at the subtropics and widened towards 